\title{
LAS PUBLICACIONES Y EL FOMENTO EDITORIAL EN LA U.D.C.A
}

\author{
Ingeborg Zenner de Polanía
}

Norella Castro Rojas

Una de las historias más conocida en el mundo, posiblemente, es acerca de la invención de la escritura y la imprenta, el desarrollo del papel y la conformación de los círculos o centros de divulgación. El libro por varios siglos, superó una serie de dificultades técnicas y de creencias culturales, como la censura, para convertirse en una herramienta indiscutible del saber humano y que hoy es una de las mercancías que tiene su rápido ascenso dentro del mundo académico, financiero, cultural, científico y artístico.

En el mundo y a través del tiempo, la academia, especialmente las Instituciones de Educación Superior, han sido promotoras del auge de la cultura escrita, del libro, mostrando la necesidad de proveer un medio de consulta, de difusión de ideas y de hallazgos científicos, pues los estudiantes, los requieren no sólo para profundizar las cátedras impartidas, sino también por la necesidad de leer los diversos autores. Así, se da inicio al desarrollo de las editoriales; la universidad pasa de ser una organización transmisora de saberes a generadora de conocimiento, herramienta pedagógica en el proceso de enseñanza-aprendizaje y cuyo fin es servir de apoyo a la docencia y a la divulgación de la producción intelectual.

La Universidad de Ciencias Aplicadas y Ambientales U.D.C.A no es ajena a este proceso, por ello, desde la edición de su primera cartilla, año de 1997, se dio a la labor de trascender el conocimiento de sus docentes y los resultados de investigaciones desarrollados en el seno de su comunidad universitaria. Su objetivo es publicar sobre la base de cuidar la necesaria calidad

1 Editora de la Revista U.D.C.A Actualidad \& Divulgación Científica.rev.udca@udca.edu.co

2 Comunicadora Social y Periodista, Coordinadora Fondo de Publicaciones U.D.C.A. norellac@udca.edu.co científica y técnica, así como la gestión, la distribución y el intercambio de libros y documentos editoriales.

A partir de este año, la U.D.C.A se dio a la tarea de incrementar sus ediciones, creando la unidad de Fondo de Publicaciones, cuyo norte es administrar el sello editorial de la Universidad y cumplir así con sus fines sustantivos de docencia, de investigación y de difusión, procurando que la diversidad temática de la producción editorial llegue a la comunidad universitaria, en particular, y al público en general, así como permitir un mayor acercamiento entre autores, editores, libreros, bibliotecas, lectores, estudiosos e investigadores. También será una instancia de apoyo que gestionará derechos de autor, impresión, distribución, promoción y comercialización. A esas funciones, se han sumado las responsabilidades derivadas de las modificaciones en la industria editorial en lo referente a las nuevas tecnologías.

Comité del fondo de publicaciones: Desde la creación del Programa de Publicaciones de la U.D.C.A, 2 de agosto de 2000, bajo la resolución No. 109, la Universidad reglamentó algunas de las actividades para la puesta en marcha del mismo; para ello, diseñó funciones y labores a realizar y organizó un equipo de trabajo, compuesto por directivos y docentes, quienes brindan asesoría y acompañamiento a todo este proceso. A partir del año 2007, estos miembros del Comité, se dieron a la tarea de definir y aprobar la normatividad editorial para la producción editorial en la Universidad. Se estimó conveniente este ejercicio, por la imperiosa necesidad de formalizar el desempeño que debe tener nuestro fondo, integrando el Manual de Estilo, como Reglamento de Publicaciones. Así mismo, estipularán las políticas editoriales, tanto para la producción de libros como para la revista científica.

Producción editorial: Después de ocho años de existencia, el Fondo de Publicaciones emprendió una nueva etapa de intensificación de sus actividades editoriales, de impulso a la edición universitaria, con la profundización 
en su presencia internacional y el desarrollo de nuevas estrategias de promoción. Este Fondo no es ni será ajeno a la innovación, pues seguirá contribuyendo a la creación del conocimiento, fortaleciendo el desarrollo social y comprometiéndose con el aporte de editar libros con calidad científica y cultural.

La producción del Fondo de Publicaciones, en sus últimos años, ascendió a 28 títulos (Figura 1), todos editados en medio impreso, algunos con soporte CD. Para este año, se tiene previsto la edición de más de cinco nuevas publicaciones y otras coediciones, mecanismo que ayuda a la promoción y difusión de las obras.
Con estos nuevos propósitos, el Comité de Publicaciones, dentro de sus políticas y con el fin de darle un ordenamiento a sus ediciones, dispondrá de una serie de colecciones, teniendo en cuenta los ejes temáticos y áreas del conocimiento y conformar su catálogo de publicaciones. Esta es una muestra de su evolución, pues día a día entiende su gestión editorial, que implica pensar, producir y difundir el libro, como un bien cultural para el desarrollo de la academia y de la sociedad. Sin embargo, debe complementar su acción, a través de otras funciones, como el estímulo a la producción intelectual en la comunidad académica, la creación de conciencia, a nivel de las autoridades universitarias, de la

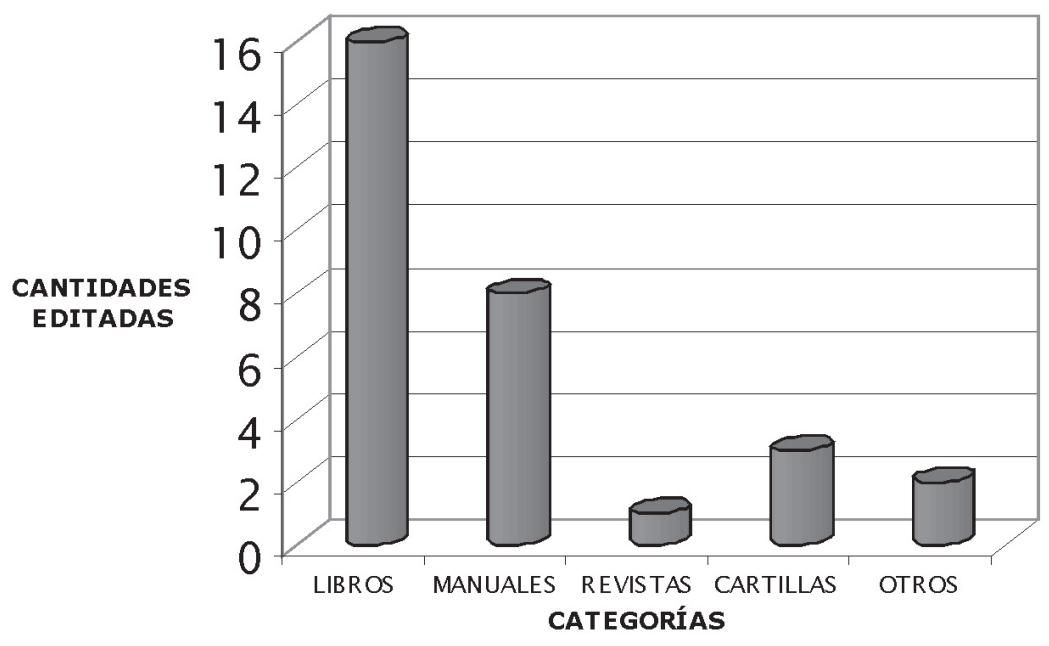

Figura 1. Tipos de publicaciones y cantidades editadas.

importancia de las editoriales universitarias, la definición de criterios comunes para mejorar sus ediciones y sus estrategias para optimizar la distribución de sus publicaciones, la participación en eventos feriales y el establecimiento de sus relaciones editoriales.

Derechos de autor: Se formalizarán instrumentos jurídicos de contratos con sus autores y de convenios, ya sean interinstitucionales o con editoriales comerciales. Otras actividades que se aprobarán en las políticas para el Fondo de Publicaciones y en común acuerdo con la Vicerrectoría Académica, la estimulación al docente para que edite sus conocimientosy, como retribución, tenga un reconocimiento en su escalafón profesoral; también compete para la participación en artículos para la Revista Científica.
La U.D.C.A, en su noble gestión de apoyo a la edición y como complemento a este incentivo, los docentesautores siempre han tenido la ventaja que la Universidad cubre el $100 \%$ de los costos de la edición de sus obras, apoyo que continuará dentro de las políticas estipuladas, como también el reconocimiento justo y acorde a su trabajo académico y de investigación, en brindarle un porcentaje, en libros y/o económico, sobre la edición.

Difusión y comercialización: La promoción del libro universitario de la U.D.C.A, se convertirá en una significativo quehacer y para ello, utilizará tácticas necesarias, como boletines de prensa, que incluyan fichas bibliográficas y reseñas, avisos en periódicos 
y otros medios de comunicación, presentaciones de libros y de colecciones a través de foros universitarios y externos, edición del catálogo de novedades, participación en eventos feriales de la cultura impresa, para que la comunidad académica, científica, cultural, conozcan la producción intelectual que emana la Universidad (Tabla 1).

La estrategia aplicada con la participación o vinculación en las redes de comercialización por Internet, especialmente en la lalibreriadelau.com, el Fondo de Publicaciones fortaleció e incrementó la venta y la difusión de sus ediciones. A través de este canal, nuestros libros son conocidos en México, Ecuador, Perú, Costa Rica, Venezuela y, recientemente, en España y Argentina. Sin un afán mercantilista y sin predominar el lucro, el Fondo ha buscado, entre muchas alternativas y continuará en ese proceso, en hacer presencia en diferentes eventos académicos del libro, que le permitan su desarrollo y volverse más eficiente y vigorosa. La U.D.C.A, como resultado de esta gestión, ha participado en las diferentes versiones de la Feria Internacional del Libro de Bogotá, con stand individual y conjuntamente con la Asociación de Editoriales Universitarias de Colombia ASEUC. Con

Tabla 1. Propuestas para la comercialización y difusión de las ediciones universitarias de la Universidad.

\begin{tabular}{|c|c|}
\hline PROPUESTA DE DIFUSIÓN & OBJETIVO \\
\hline Institucionalizar el sello editorial & $\begin{array}{l}\text { Crear un logotipo que identifique al Fondo de Publicaciones e institucionalizarlo, a nivel } \\
\text { interno y externo. }\end{array}$ \\
\hline Seleccionar la presentación de las publicaciones & $\begin{array}{l}\text { Fijar una política para la edición final de las publicaciones, es decir, el tipo de formato, } \\
\text { teniendo en cuenta el impacto que tendrá la obra y su público primario y secundario. }\end{array}$ \\
\hline $\begin{array}{l}\text { Fijar una visión sobre el proceso de mercado y de } \\
\text { comercialización }\end{array}$ & $\begin{array}{l}\text { Es decir, orientar a los docentes-autores en qué áreas, géneros o temas se puede } \\
\text { incursionar y que aporten al estudiante y a la sociedad y su circulación y/o visualización } \\
\text { sea de impacto. Esto garantiza la calidad, la conveniencia y la pertinencia de las } \\
\text { publicaciones. }\end{array}$ \\
\hline $\begin{array}{l}\text { Fortalecer y definir canales de distribución y de } \\
\text { comercialización }\end{array}$ & $\begin{array}{l}\text { Diseñar y gestionar estrategias efectivas de mercadeo y promoción, para el desarrollo } \\
\text { permanente de la labor del Fondo. }\end{array}$ \\
\hline $\begin{array}{l}\text { Organizar y conformar la librería universitaria dentro } \\
\text { del Campus Universitario }\end{array}$ & $\begin{array}{l}\text { Es una labor a largo plazo, pero es una de las estrategias complementarias tanto para } \\
\text { la difusión como para la comercialización del libro, pues se tiene vínculo directo con el } \\
\text { comprador, ya sea estudiante, libreros, distribuidores. }\end{array}$ \\
\hline $\begin{array}{l}\text { Ampliar la gama de servicios editoriales y temas } \\
\text { de publicación }\end{array}$ & $\begin{array}{l}\text { Tener acceso a ruedas de negocios de traducciones, de compra de derechos de autor, } \\
\text { de coediciones internacionales. }\end{array}$ \\
\hline $\begin{array}{l}\text { Conformar un grupo de trabajo dedicado a la } \\
\text { edición }\end{array}$ & $\begin{array}{l}\text { Este equipo debe incluir diseñadores, evaluadores, correctores de estilo y comercializadores, } \\
\text { entre otros. }\end{array}$ \\
\hline Elaborar un catálogo de publicaciones & $\begin{array}{l}\text { Herramienta de comercialización y de difusión, que contará con una serie de colecciones } \\
\text { y la cual se podrá implementar en diferentes eventos feriales, tanto a escala nacional } \\
\text { como internacional. }\end{array}$ \\
\hline $\begin{array}{l}\text { Crear una cultura institucional contra la fotocopia } \\
\text { y la piratería }\end{array}$ & $\begin{array}{l}\text { A través del Centro Colombiano de Derechos Reprográficos CEDER, concienciar tanto al } \\
\text { estudiante como al docente sobre este tema. }\end{array}$ \\
\hline $\begin{array}{l}\text { Crear una política de participación en eventos } \\
\text { feriales }\end{array}$ & $\begin{array}{l}\text { Participar, en la medida de lo posible, en todos los eventos feriales del libro, ya sean } \\
\text { regionales, nacionales e internacionales. }\end{array}$ \\
\hline $\begin{array}{l}\text { Programar actividades académicas y culturales que } \\
\text { tengan que ver con el mundo del libro }\end{array}$ & $\begin{array}{l}\text { Conversatorios sobre Derechos de Autor, Talleres sobre Producción Editorial, dirigidos } \\
\text { tanto para docentes, como para administrativos. }\end{array}$ \\
\hline
\end{tabular}


gran éxito, en las instalaciones del Campus Universitario, logró el montaje de tres Ferias del Libro Universitario, con el firme propósito de instalar la cuarta, en el mes de agosto de 2008, en el marco de los 25 años de la Universidad.

La gestión y el apoyo administrativo: Para llevar a cabo todas los retos propuestas y bajo la reglamentación de las nuevas políticas editoriales, en el artículo titulado Propuestas de Reestructuración del Fondo de Publicaciones de la U.D.C.A, editado en la Revista U.D.C.A Actualidad \& Divulgación Científica, Año 10 No. 2, se propuso como gestión administrativa, entre otros:

- Obtención de su plena autonomía administrativa y financiera, con el apoyo irrestricto de la rectoría y aquellas unidades que tengan que ver directamente con la labor del Fondo de Publicaciones.

- Determinar las líneas editoriales en Ciencias de la Salud, Arte, Medicina Veterinaria, Administración, Enfermería, Pedagogía, entre otras, mediante la publicación y difusión de obras con valor científico, histórico, cultural, pedagógico, artístico o literario.

- Crear el Comité Académico para cada una de las facultades, asignándole responsabilidades y consolidar las funciones referentes a las ediciones.

Varios autores, especialistas en el tema de las Editoriales Universitarias en el mundo, coinciden que la producción editorial de las universidades debe enfrentar nuevos desafíos, cada vez más exigentes, académica y socialmente, en el cumplimiento ético de la calidad pedagógica y científica, retos que también persigue y establece la Universidad y que debe irrumpir en los recientes y estrepitosos cambios tecnológicos y comerciales para su progreso.

\section{REVISTA U.D.C.A ACTUALIDAD \& DIVULGACIÓN CIENTÍFICA}

Desde su creación, hace 10 años, la Revista U.D.C.A Actualidad $\mathcal{E}$ Divulgación Científica ha tenido como finalidad hacer visible a la comunidad académica y científica los productos de las investigaciones realizados por el cuerpo docente de las diversas facultades de la Universidad y divulgar los hallazgos proporcionados por investigaciones ejecutadas en otros organismos nacionales o internacionales y, en general, de la sociedad.
Se pretendió editar una revista que brinde al público técnico y científico una publicación multidisciplinaria, que comprendiera estudios básicos y aspectos relacionados con el sector salud, el sector agropecuario, el sector social-humanístico y el sector económico.

Actualmente, la revista edita dos fascículos anuales, y abarca artículos técnicos o de revisión, artículos de reflexión y artículos científicos, evaluados por árbitros especialistas en el tema. Es indexada en el Chemical Abstracts Services, CAS (Columbus, Ohio, USA); en el $\mathrm{CAB}$ Abstracts (Inglaterra) y en Publindex (Colombia), en esta última, en categoría $\mathrm{B}$, y reseñada en Periódica (UNAM, México). No siempre fue así, ni fue fácil cumplir estos objetivos, pero se logró, finalmente, posesionarla. A medida que los proyectos de investigación, tanto formativos como aquellos propuestos por los profesores, aumentaban en la U.D.C.A y su ejecución proporcionaba resultados publicables, la revista incursionó en la evaluación por pares e incluyó todos los aspectos que caracterizan una publicación científica.

Durante los primeros cinco años de existencia de la revista, no se contaba con su aceptación como medio para hacer visible los resultados de investigaciones por parte de los docentes de la U.D.C.A, ni tampoco se había posesionado la publicación en el ámbito académico nacional. No existían normas e instrucciones para los autores, los cuales, se implementaron a partir de la entrega del volumen 4, No. 2 (junio -diciembre de 2003); estas siguen vigentes con algunas pocas adiciones, ampliaciones y correcciones. Contempla, el instructivo, la publicación de tres tipos de textos que, de acuerdo a PUBLINDEX, corresponden a las siguientes tipologías: artículo de investigación científica y tecnológica, de reflexión y de revisión.

El propósito principal de la segunda etapa, 20032007, de la Revista fue lograr la indexación nacional e internacional. Esto, basado en la estabilidad, en lograr que la publicación se tornara atractiva para la comunidad de investigadores externa a la universidad y en hacer mucho más visible los artículos publicados, ante todo, por o con la colaboración de los árbitros externos, expertos en los diversos temas que abarca nuestra revista multidisciplinaria. Este empeño, se logró plenamente y después de varios intentos e indexaciones en la categoría C, en noviembre del año 2007, la revista fue indexada en la categoría B, de PUBLINDEX. 
A continuación, se muestran y se analizan los aspectos relevantes de los últimos cinco años que condujeron al reconocimiento de la calidad de la revista.

Árbitros externos: A partir de la segunda entrega del año 2004, volumen 7, No. 2, se contó, además de los árbitros internos, con la desinteresada colaboración de expertos externos, quienes evaluaron los textos sometidos. Para la escogencia de estos árbitros, se aprovechó el servicio de búsqueda de "evaluadores pares" proporcionado por COLCIENCIAS, conjuntamente con el análisis de la hoja de vida de los expertos, disponibles en el CvLac.

Aquí, se quiere destacar la adecuada y comprometida aportación de los profesionales colaboradores. Hasta el momento hemos contado con 107 expertos nacionales, cumplidores de los requisitos y exigencias de PUBLINDEX. El mayor número de árbitros correspondió a docentes investigadores de la Universidad Nacional, sedes Bogotá, Medellín y Palmira. Le sigue, la Universidad de Antioquia, la Pontificia Universidad Javeriana, la Universidad de Bogotá Jorge Tadeo Lozano, la Universidad del Valle, la Universidad de Córdoba y la Universidad Tecnológica y Pedagógica de Tunja. Aquí es imposible mencionar a todas las 31 universidades y profesores de todos los departamentos del país, quienes han acompañado a nuestra revista, como tampoco podemos destacar a todos las otras instituciones donde laboran nuestros pares evaluadores. En cada número impreso, se hace un sencillo reconocimiento a cada uno de los árbitros, publicando su nombre. Igualmente han sido evaluados textos por árbitros extranjeros, investigadores de la Universidad de California, las Universidades Federales Brasileras de Sao Carlos y Minas de Gerais, la Universidad de Buenos Aires y el Schumacher College, de Inglaterra.

Tipología de documentos publicados: Cada número de la revista muestra, como primera medida, el editorial, a cargo del señor rector de la Universidad o, excepcionalmente, por otro dignatario de la U.D.C.A. El tema escogido versa sobre algún aspecto relevante de la academia, como, por ejemplo, la mundialización de la educación superior, la ciencia y la tecnología vista desde el interior de la universidad y la relación con el sector privado.

Luego, siguen los otros documentos, catalogados de acuerdo a su tipología y su tema. Como se puede observar en la Figura 2, a partir del año 2003, predominaron los artículos científicos sobre aquellos de otro tipo.

Seguir con esta tendencia es un quehacer complejo y difícil de cumplir, ante todo por la renuencia de algunos autores de aceptar estas tipologías, las cuales requieren mayores esfuerzos y empeños que solamente se derivan de los resultados de proyectos de investigación terminados.

Contribuciones de autores de la U.D.C.A: Uno de los propósitos de una revista universitaria es proporcionar a los docentes-investigadores un medio confiable y eficiente

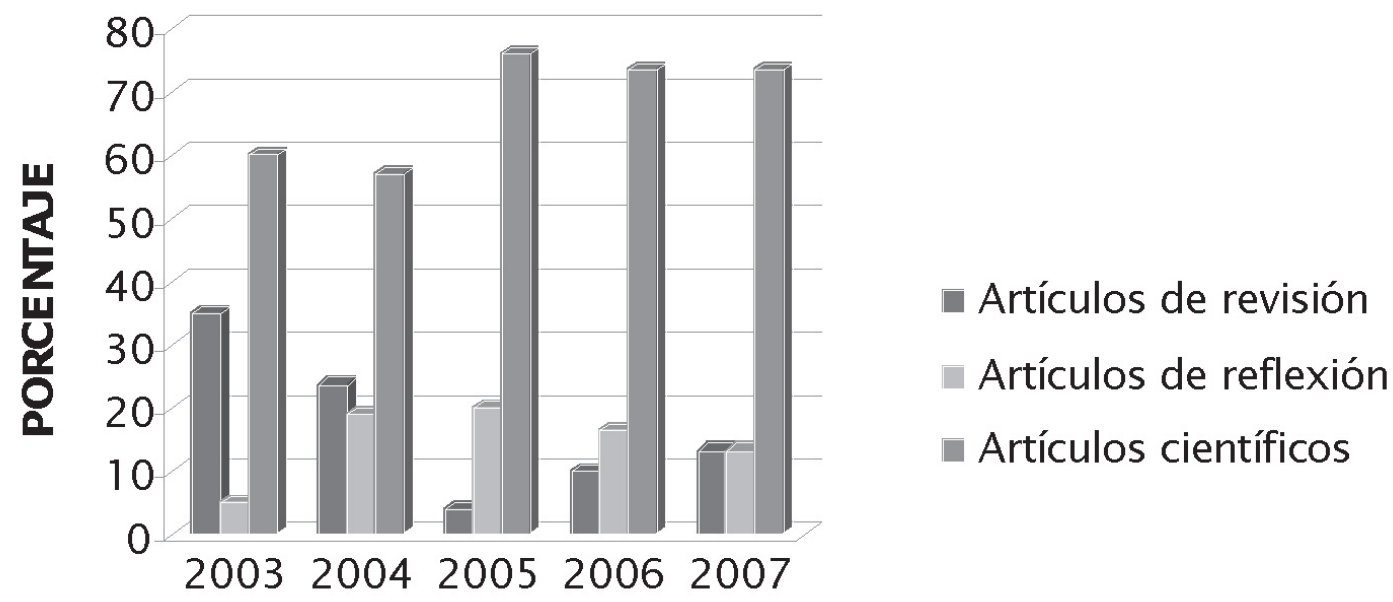

Año

Figura 2. Relación de artículos publicados en la tipología 1), 2) y 3), establecida por PUBLINDEX. 
para hacer visible los resultados de sus investigaciones y para recibir, a través de los árbitros externos, a menudo docentes de mayor experiencia y trayectoria de investigador y publicador, consejos y sugerencias positivos, para mejorar y avanzar como investigador.

En la tabla 2, se consignan los artículos publicados y presentados por los docentes de las facultades de la U.D.C.A. Se observa, en general, que aquellas con mayor edad cronológica poseen también una mayor trayectoria investigativa y, por ende, una amplia productividad intelectual.
Participación de autores de otras universidades y centros de investigación: La contribución con textos de autores externos a la U.D.C.A, se inició en el año 2004, con la aparición de la Revista, en la lista de Publicaciones Científicas Indexadas en Colombia. De allí en adelante, como se puede deducir de la figura 3, el número de artículos publicados, de origen externo, está en continuo aumento.

En el año 2004, en el fascículo 7(1), se publicó el primer artículo procedente de otra universidad: la Pontificia Para el siguiente año, además de las universidades mencionadas, enviaron sus contribuciones, autores

Tabla 2. Contribución por facultad U.D.C.A, expresada en artículos publicados en los últimos cinco años.

\begin{tabular}{|c|c|c|c|c|c|c|}
\hline AÑO & $\mathbf{2 0 0 3}$ & $\mathbf{2 0 0 4}$ & $\mathbf{2 0 0 5}$ & $\mathbf{2 0 0 6}$ & $\mathbf{2 0 0 7}$ & TOTAL \\
\hline FACULTAD & 0 & 2 & 2 & 1 & 1 & 6 \\
\hline Medicina & 0 & 0 & 1 & 1 & 1 & 3 \\
\hline Enfermería & 0 & 0 & 1 & 0 & 0 & 1 \\
\hline Ciencias del Deporte & 6 & 3 & 2 & 4 & 2 & 17 \\
\hline Medicina Veterinaria & 2 & 0 & 0 & 1 & 0 & 3 \\
\hline Med. Vet. y Zootecnia & 0 & 1 & 1 & 1 & 0 & 3 \\
\hline Zootecnia & 1 & 2 & 1 & 3 & 1 & 8 \\
\hline Ingeniería Comercial & 0 & 1 & 1 & 0 & 0 & 2 \\
\hline Ing. Geogr. y Amb. & 3 & 6 & 4 & 6 & 5 & 24 \\
\hline Ingeniería Agronómica & 1 & 0 & 0 & 1 & 1 & 3 \\
\hline Ciencias Básicas & 0 & 2 & 1 & 0 & 1 & 4 \\
\hline Otras dependencias & & & & & & \\
\hline
\end{tabular}

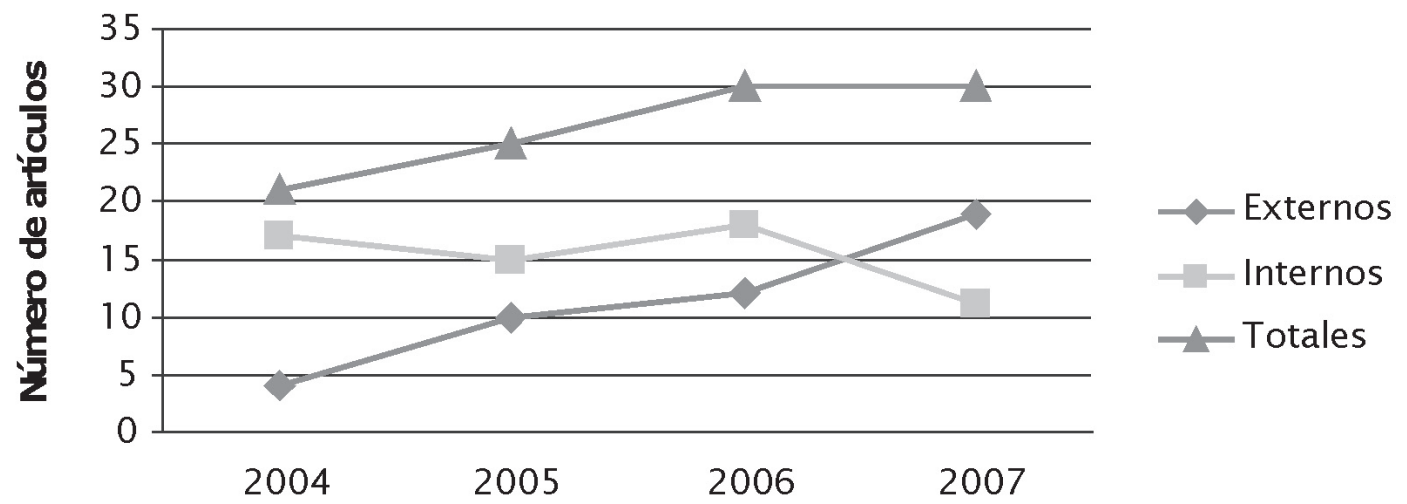

Años

Figura 3. Procedencia de artículos publicados en los últimos cuatro años de existencia de la revista. 
de las Universidades del Magdalena, Nacional de Colombia, UPTC de Tunja, Los Andes, Jorge Tadeo Lozano y CENICAFÉ. En los años sucesivos, se sumaron la Universidad Pedagógica Nacional, Universidad del Tolima, Universidad del Rosario, CENIPALMA y la Secretaría de Salud del Meta, conjuntamente con la Universidad de los Llanos.

Resumiendo, se observa que del año 2004 hasta la fecha, el 38,68\% de los artículos tenía como autores a investigadores externos a la U.D.C.A; PUBLINDEX exige un $30 \%$, ya que considera que una publicación indexada no solamente debe publicar textos internos, sino manifestar el interés que científicos externos demuestran por la publicación. Muchos investigadores, que no pertenecen a la Universidad, han considerado una publicación seriada multidisciplinaria, como la nuestra, de mayor impacto para las comunidades académicas que las revistas especializadas.
Futuro: Las metas a lograr en el próximo quinquenio de la Revista corresponden a seguir mejorando la calidad científica y, basado en ella, ser reconocida como un medio codiciado de hacer visible los hallazgos derivados de investigaciones. Se pretende ampliar la aparición de la revista a tres fascículos por año. Además, se recibirán textos en un segundo idioma, como por ejemplo inglés y portugués, extendiendo así la cobertura internacional.

Finalmente, se buscará la indexación en la categoría A2 de PUBLINDEX. Con el requisito de que "el $50 \%$ de los miembros del comité científico, debe pertenecer a instituciones extranjeras", se cumplió; disponemos, a partir de esta entrega, de la colaboración de científicos - investigadores de República Dominicana, del Brasil, del Ecuador, de Venezuela, de Norteamérica, de España y de México.

Recibido: Enero 20 de 2008

Aceptado: Mayo 13 de 2008 